\title{
Quantitative Characterization of Misfit Dislocations at GaP/Si Heteroepitaxial Interfaces via Electron Channeling Contrast Imaging and Semi-Automated Image
} Analysis

\author{
Ari N. Blumer ${ }^{1}$, Jacob T. Boyer ${ }^{1}$, Julia I. Deitz ${ }^{1}$, Francisco A. Rodriguez ${ }^{1}$ and Tyler J. Grassman ${ }^{1,2^{*}}$ \\ 1. Dept. of Materials Science \& Engineering, The Ohio State University, Columbus, OH, USA. \\ 2. Dept. of Electrical \& Computer Engineering, The Ohio State University, Columbus, OH, USA. \\ * Corresponding author: grassman.5@osu.edu
}

Characterization of crystalline defects in semiconducting materials is crucial for the development of wellinformed growth methods and ultimately high-performance optoelectronic devices. An excellent example is the technologically important, but fundamentally complex, heteroepitaxial $\mathrm{GaP} / \mathrm{Si}$ system. GaP/Si is presently under extensive consideration as an enabling materials integration pathway for the production of III-V/Si photovoltaics and optoelectronics. However, due to lattice-mismatch and interfacial heterovalency, $\mathrm{GaP} / \mathrm{Si}$ growth can result in a multitude of extended defects (e.g. misfit dislocations), the control of which is critical for the performance of associated devices. To achieve the resolution necessary for characterization of such defects, transmission electron microscopy (TEM) has generally been accepted as the most viable technique. However, the extensive sample preparation required, and the relatively small resultant sample areas, can lead to characterization bottlenecks and insufficiently representative results. An alternative diffraction imaging technique, electron channeling contrast imaging (ECCI) [1]-[3], which is growing in popularity within the semiconductor epitaxy field, provides a number of inherent advantages over TEM [4]-[7]. Performed in a scanning electron microscope (SEM), ECCI enables high-throughput, non-destructive characterization of surface and subsurface defects across much larger specimens with little to no sample preparation. With ECCI it is possible to rapidly collect extremely large image sets, from which statistically relevant quantitative descriptors can nominally be extracted, enabling the determination of key defect formation and evolution properties, like dislocation dynamics. To this end, we briefly describe preliminary work on quantitative ECCI-based characterization of misfit dislocations at the $\mathrm{GaP} / \mathrm{Si}$ interface using semi-automated image analysis, with the ultimate goal of providing detailed insight into the dislocation dynamics in this model system.

Depending on the epitaxial processes used to produce the GaP/Si structures, the range and morphology of the resulting misfit dislocation networks can vary greatly, with densities and line lengths spanning orders of magnitude (Fig. 1). Statistically relevant quantification thus requires the use of large total image areas $\left(0.1 \mathrm{~mm}^{2}\right.$ or more). In such cases, manual image analysis is highly impractical, if not effectively impossible. Therefore, we are investigating both state-of-the-art segmentation analysis, as well as machine learning approaches, toward the development of a robust set of semi-automated image analysis procedures for the extraction of a wide range of misfit dislocation statistics, which can be compared against and/or used to develop descriptive and predictive dislocation dynamics models. To date, we have had the most success with the MIPAR image analysis package [8] for batch-processed segmentation of very large area ECCI micrographs and montages. This method has yielded reliable and accurate (within 5\% of manual analysis) extraction of complete histograms (Fig. 2) and subsequent statistics of values such as misfit dislocation length, line density, and Burgers vector versus line direction and epitaxial process conditions. Moving forward, we continue to refine our segmentation procedures, as well as explore machine learning techniques to assist in overcoming some of the limitations encountered in traditional segmentation 
analysis. We will thus discuss our current status in both the image analysis and resultant statistical dislocation analysis.

\section{References:}

[1] MA Crimp, Microsc. Res. Tech. 69 (2006), p. 374.

[2] AJ Wilkinson, J. Electron Microsc. (Tokyo) 49 (2000), p. 299.

[3] S Zaefferer and N-N Elhami, Acta Mater. 75 (2014), p. 20.

[4] YN Picard et al., Appl. Phys. Lett. 91 (2007), p. 094106.

[5] G Naresh-Kumar et al., Phys. Rev. Lett. 108 (2012), p. 135503.

[6] JI Deitz et al., J. Vis. Exp. 101 (2015), p. e52745.

[7] K Mukherjee et al., Appl. Phys. Lett. 110 (2017), p. 232101.

[8] JM Sosa et al., Integrating Mater. Manuf. Innov. 3 (2014), p. 10.
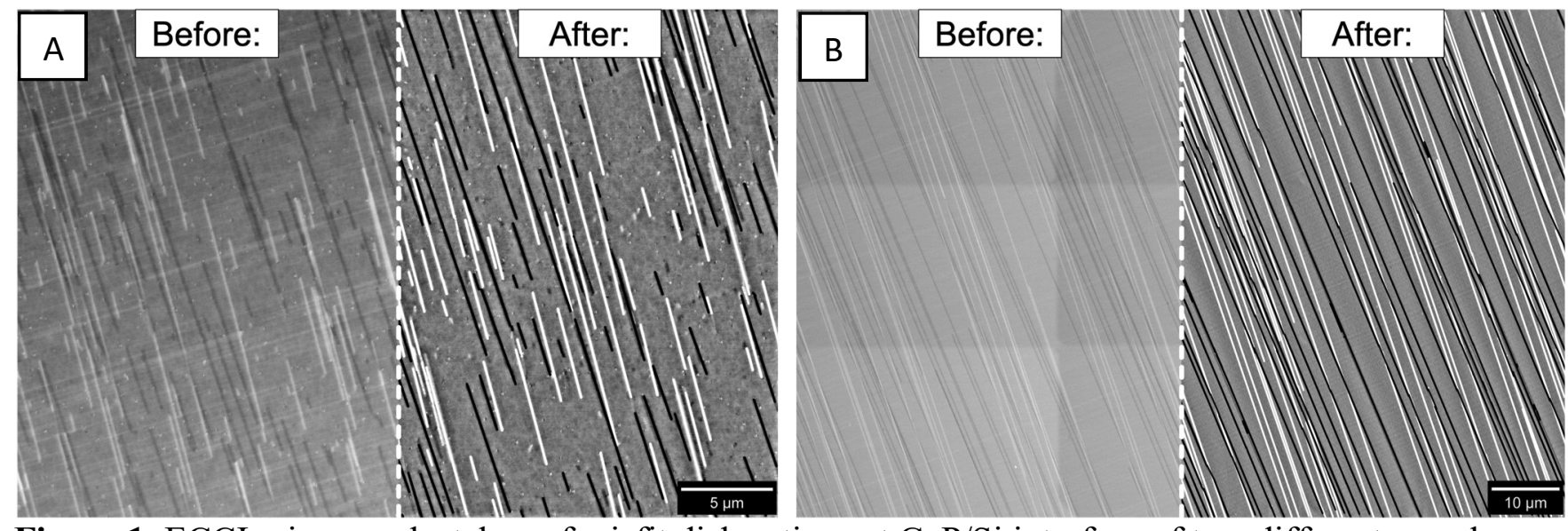

Figure 1. ECCI micrographs taken of misfit dislocations at $\mathrm{GaP} / \mathrm{Si}$ interface of two different samples grown with different epitaxial process conditions, showing before and after image processing and segmentation, where A) is a single image and B) is a $3 \times 3$ stitched image

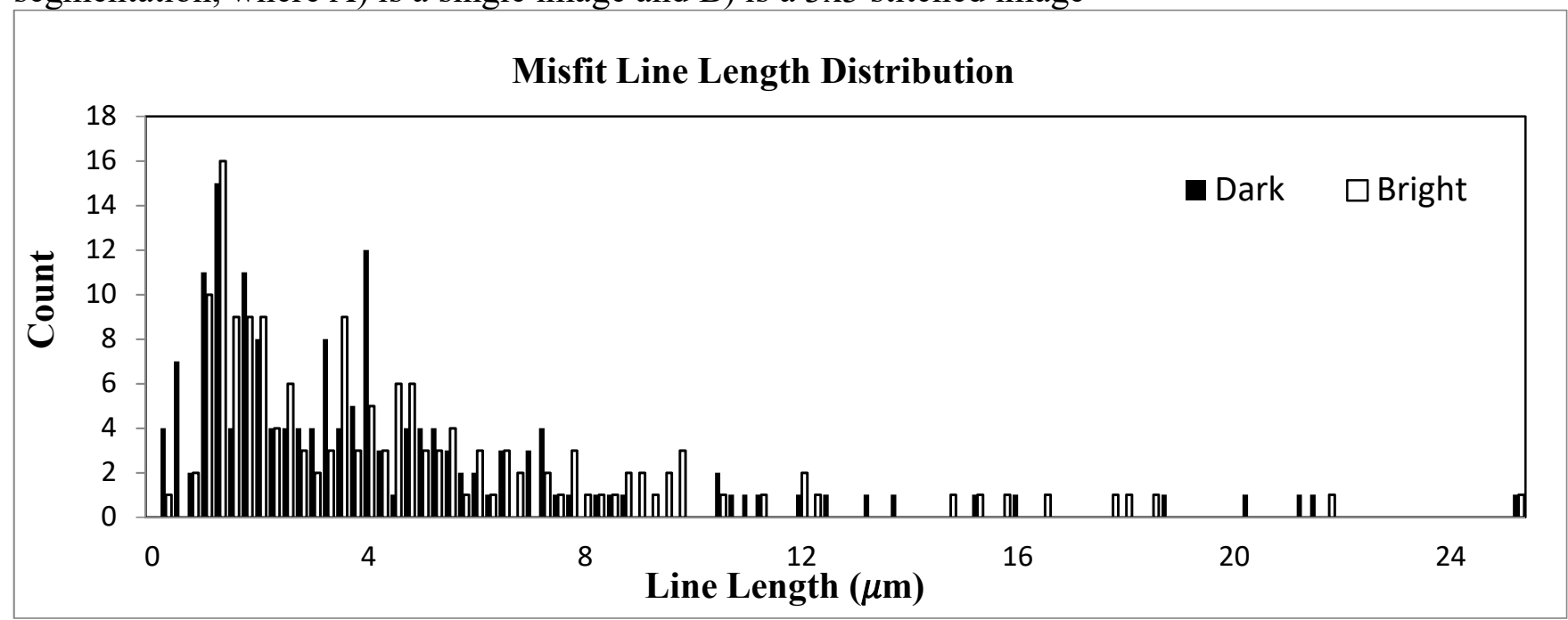

Figure 2. An example histogram of the line length distribution of misfit dislocations using data directly from the segmentation analysis performed on the ECCI micrograph in Fig. 1A. 